\title{
Análisis de la implantación del Grado de Economía y Administración y Dirección de Empresas a través de su metodología exclusivamente online.
}

María Consuelo Calafat Marzal, José Manuel Guaita Martínez, María Luisa Martí Selva y Rosa María Puertas Medina.

chelo@esp.upv.es, josemanuel.guaita@campusviu.es, mlmarti@esp.upv.es, rpuertas@esp.upv.es.

\begin{abstract}
Valencia International University (VIU) taught her Degrees and Masters Degrees through a virtual training system using a method of videoconferencing. All this is done through shared rooms in a platform called "blackboard". The objective of the work presented is to analyze the process of implementing Degrees of Economics and Business Administration (ADE) started in the academic year 2014-15. They have been used as learning tools forums, guided activities, seminars, videoconferencing, presentation of video works by students and watching movies. It is quantitatively and qualitatively assess the degree of student learning, using such techniques as the studentteacher relationship evolves into a fully virtual environment, using all the potential provided by the Information and Communication Technologies (ICT). The results have shown that students are able to adapt quickly to new learning methodologies achieving a mastery of computer platform. Learning is assessed continuously using the forums, guided activities, viewed and sent videos, seminars offered and the doubts raised and resolved.
\end{abstract}

Keywords: Online, Degrees Economics and Business Administration, guided activities, videoconferences, seminars, competitions, forums. 
Análisis de la implantación del Grado de Economía y Administración y Dirección de Empresas a través de su metodología exclusivamente online.

\section{Resumen}

La Universidad Internacional de Valencia (VIU) imparte sus Grados y Masters a través de un sistema de formación virtual utilizando un procedimiento de videoconferencias. Todo ello se realiza mediante salas compartidas dentro de una plataforma denominada "blackboard". El objetivo del trabajo que se presenta es analizar el proceso de la implantación de los Grados de Economía, y Administración y Dirección de Empresas (ADE) comenzado en el curso académico 2014-15. Se han utilizado como herramientas de aprendizaje los foros, las actividades guiadas, los seminarios, las videoconferencias, la presentación de trabajos en vídeo por parte de los alumnos y el visionado de películas. Se trata de valorar cuantitativa y cualitativamente el grado de aprendizaje de los alumnos, pues mediante estas técnicas la relación alumno-profesor se desenvuelve en un entorno integralmente virtual, aprovechando toda la potencialidad facilitada por las Tecnologías de Información y Comunicación (TIC). Los resultados han demostrado que los alumnos son capaces de adaptarse con rapidez a las nuevas metodologías de aprendizaje logrando un gran dominio de la plataforma informática. El aprendizaje se valora de forma continua utilizando los foros, las actividades guiadas, los vídeos vistos y enviados, los seminarios ofrecidos y las dudas planteadas y resueltas.

Palabras clave: Online, Grados Economía y Administración y Dirección de Empresas, actividades guiadas, videoconferencias, seminarios, competencias, foros, TIC. 


\section{Introducción}

La Universidad Internacional de Valencia (VIU) ha implantado en el curso 2014-15 los grados de Economía (GE) y grados de Administración y Dirección de Empresas (GADE). Dentro del plan estratégico impulsado por su máximo accionista (Grupo Planeta) ha incluido estos dos grados dentro de la enseñanza online y audiovisual que caracteriza a todos los Grados y Masters impartidos en la VIU.

La VIU ha ofertado este curso académico un total de 6 Grados y 8 Másteres, utilizando las Tecnologías de la Información y la Comunicación como piedra angular de la innovación educativa (Reyes y Chaparro, 2013). Esta universidad cuenta con un Campus Virtual con acceso desde cualquier lugar a través de un ordenador, la metodología incluye el uso de un software de videoconferencia con el objetivo de permitir al alumnado y al profesorado la interacción en vivo y en directo.

Esta instrumentación online está fundamentada en la tecnología de la plataforma Blackboard, desarrollada por Blackboard Inc., una compañía estadounidense que cotiza en el NASDAQ y está especializada en el aprendizaje en línea (e-learning) y desarrollo de programas empresariales y comercio electrónico (e-commerce). En nuestro caso, es la herramienta que facilita el acceso de las innovaciones educativas en cualquier lugar mediante la conexión personas - tecnología. Cada vez son más instituciones y docentes quienes incorporan el uso de las plataformas virtuales para la docencía y la evaluación continua, con la generación de un nuevo entorno social de aprendizaje (Gil, 2015)

El objetivo del artículo es presentar la implantación de los GE y GADE en el primer curso, teniendo en cuenta que 9 asignaturas de cada Grado comparten contenidos.

Es importante destacar el peso que en la técnica online, utilizada en la VIU, se otorga a las videoconferencias bidireccionales. Se trata del mecanismo de comunicación docentealumno más común empleado para impartir la mayoría de las clases, seminarios, tutorías individuales y grupales, actividades guiadas, entre otros. El estudiante dispone de una guía docente, explicada por el tutor tanto al inicio del módulo como al comienzo de cada asignatura, ésta sirve para orientar su trabajo y dedicación temporal. También se le facilitada un material básico de trabajo para el estudio de todas las materias.

En el trabajo que se presenta se ha analizado el uso de las herramientas online a disposición de los alumnos, su capacidad de adaptación al estudio de las asignaturas de los Grados de ADE y Economía a través de este entorno virtual que implica tanto el dominio de la plataforma "blackboard" como la tecnología ofrecida en conjunto las TIC (foros, vídeos, correos, seminarios, actividades guiadas y videoconferencias). Se ha medido dicho dominio a raíz de las respuestas realizadas por los propios alumnos en cuanto al tiempo empleado y al contenido enviado por los alumnos en base a las preguntas y casos propuestos.

(c)) EY-NC-ND 2015, Universitat Politècnica de València

Congreso In-Red (2015) 
Análisis de la implantación del Grado de Economía y Administración y Dirección de Empresas a través de su metodología exclusivamente online.

\section{Metodología de aprendizaje y desarrollo de la innovación}

Cada alumno dispone de un espacio personal de tutorías, realizándose a través de videoconferencias, chats y correo electrónico, en función de las necesidades específicas y del desarrollo personal del trabajo. El tutor de cada asignatura ha sido el encargado de programar las actividades conjuntas y gestionar el adecuado aprovechamiento del alumno. Además, ha propuesto, revisado y valorado las tareas, hayan sido realizadas como trabajo individual o en equipo, ampliadas o centradas en aspectos específicos. En el caso de estos Títulos el manejo de las herramientas virtuales y las diferentes plataformas, así como los mecanismos informáticos, ha conllevado un valor añadido a la formación de los estudiantes (Reyes y Chaparro, 2013).

El curso se planteó con las siguientes características

- Metodología online

- 10 Asignaturas por Grado de 6 ECTS, 5 para el primer cuatrimestre y 5 para el Segundo.

- $\quad$ Tiempo de impartición por asignatura: 4-5 semanas

Cada asignatura tiene la siguiente estructura:

- 1 Tutoria Colectiva de Presentación

- 3 Videoconferencias

- 2 Seminarios

- 3 Actividades Guiadas

- 1 Tutoría Colectiva Final

Todas las sesiones anteriormente descritas quedan grabadas en el Campus Virtual pudiendo ser visualizadas por el alumno en cualquier momento. La herramienta del Blackboard permite visualizar los contenidos al mismo tiempo que el alumno puede ver al profesor explicando el contenido de las diapositivas tal como se observa en la Figura 1. Esta herramienta permite mostrar el contenido de cualquier documento que se quiera compartir y en todo tipo de formato (.doc,.pdf,.xls,.pptx,etc) y además contemplar cualquier página web que el profesor crea conveniente siguiendo todos los pasos marcados por el docente. Los alumnos pueden pedir la palabra para realizar cualquier pregunta en tipo real, en esta acción tienen capacidad para elegir ser o no visionados, ser oidos a través del micrófono de su ordenador o elegir simplemente el mensaje escrito a través del chat.

(c) EY-NC-ND 2015, Universitat Politècnica de València

Congreso IN-RED (2015) 


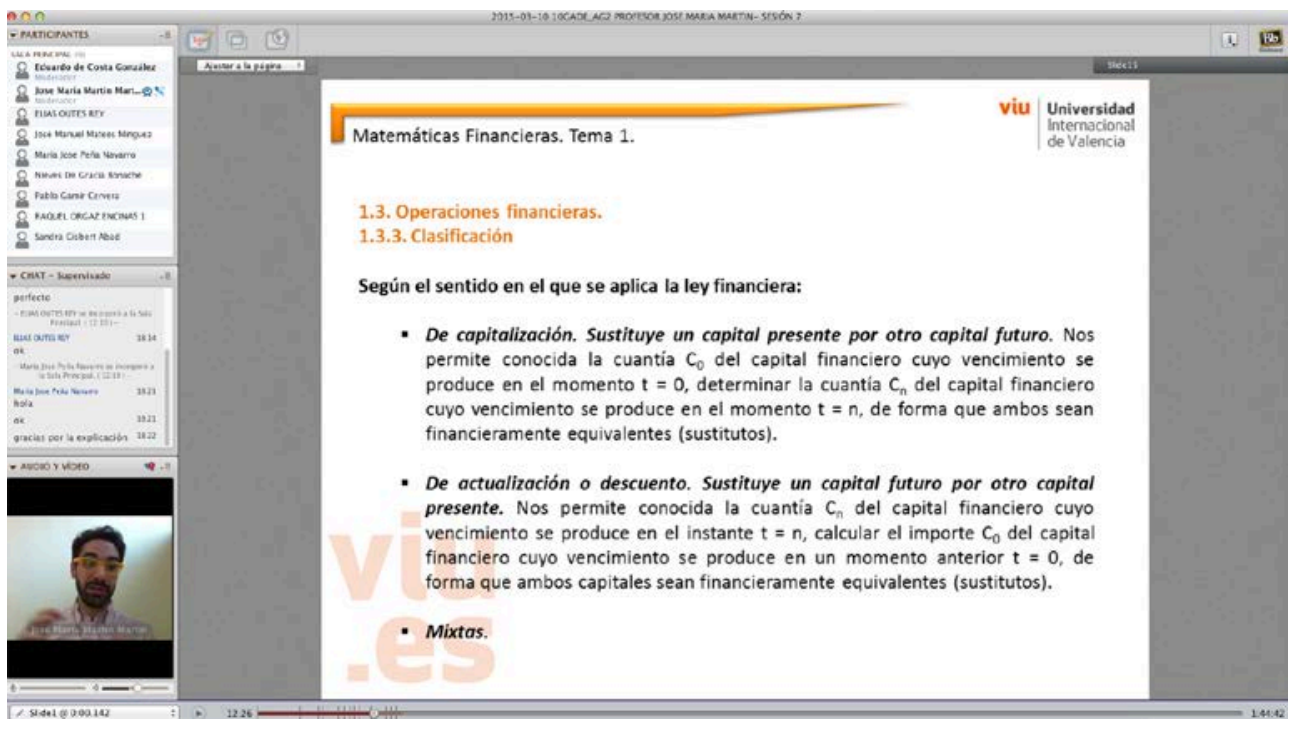

Fig. 1 Ejemplo de asignatura impartida a través del BlackBoard

Los alumnos de las distintas asignaturas son evaluados de forma continua a través de las actividades y trabajos que los profesores solicitan a sus alumnos. Además cuando finaliza el periodo docente tienen una prueba presencial en las sedes de la VIU de las principales ciudades de cada país, según los alumnos matriculados.

\section{Resultados}

En este primer año del GE y GADE se han realizado 182 matriculaciones de asignaturas, correspondientes a 30 alumnos, 10 en el GE y 20 para el GADE.

El curso se ha desarrollado exclusivamente online con lo que ha sido necesario explicar en las primeras clases la importancia de la disciplina a la hora de la lectura de los contenidos y el seguimiento de las sesiones impartidas en videoconferencia. Esta labor ha sido reforzada por el profesorado medinate envio de correos y anuncios recordando el calendario de sesiones y actividades guiadas.

Esta metodología requiere que los alumnos reciban una formación inicial sobre el uso y las características de la plataforma Blackboard, así como videos explicativos a los cuales tienen acceso para reforzar el conocimiento de la tecnología educativa que están utilizando. El $100 \%$ de los alumnos ha accedido a la plataforma, a los contenidos, a las sesiones grabadas y al envio de las actividades realizadas, con relativa facilidad. La VIU tiene un departamento de atención al alumno para solucionar todas las cuestiones y problemas

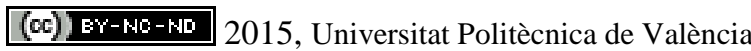

Congreso In-Red (2015) 
Análisis de la implantación del Grado de Economía y Administración y Dirección de Empresas a través de su metodología exclusivamente online.

tecnológicos surgidos al principio del curso. Los alumnos terminan dominando la plataforma informática, convirtiéndose en su herramienta de estudio.

A continuación se analizan los trabajos solicitados por parte de cada profesorado para la evaluación continua del alumnado, tanto en plazo como en contenido de la misma como eje fundamental del presente estudio. Tal como se indica en la Figura 2 y Tabla 1, las actividades guiadas han sido presentadas en el plazo exigido a través de un portfolio personalizado, siendo un elemento muy relevante en la gestión de las calificaciones (Escámez et al.,2010). De esta forma el profesor y el alumno tienen acceso al contenido de la práctica, a la nota y a los comentarios realizados por el profesor.

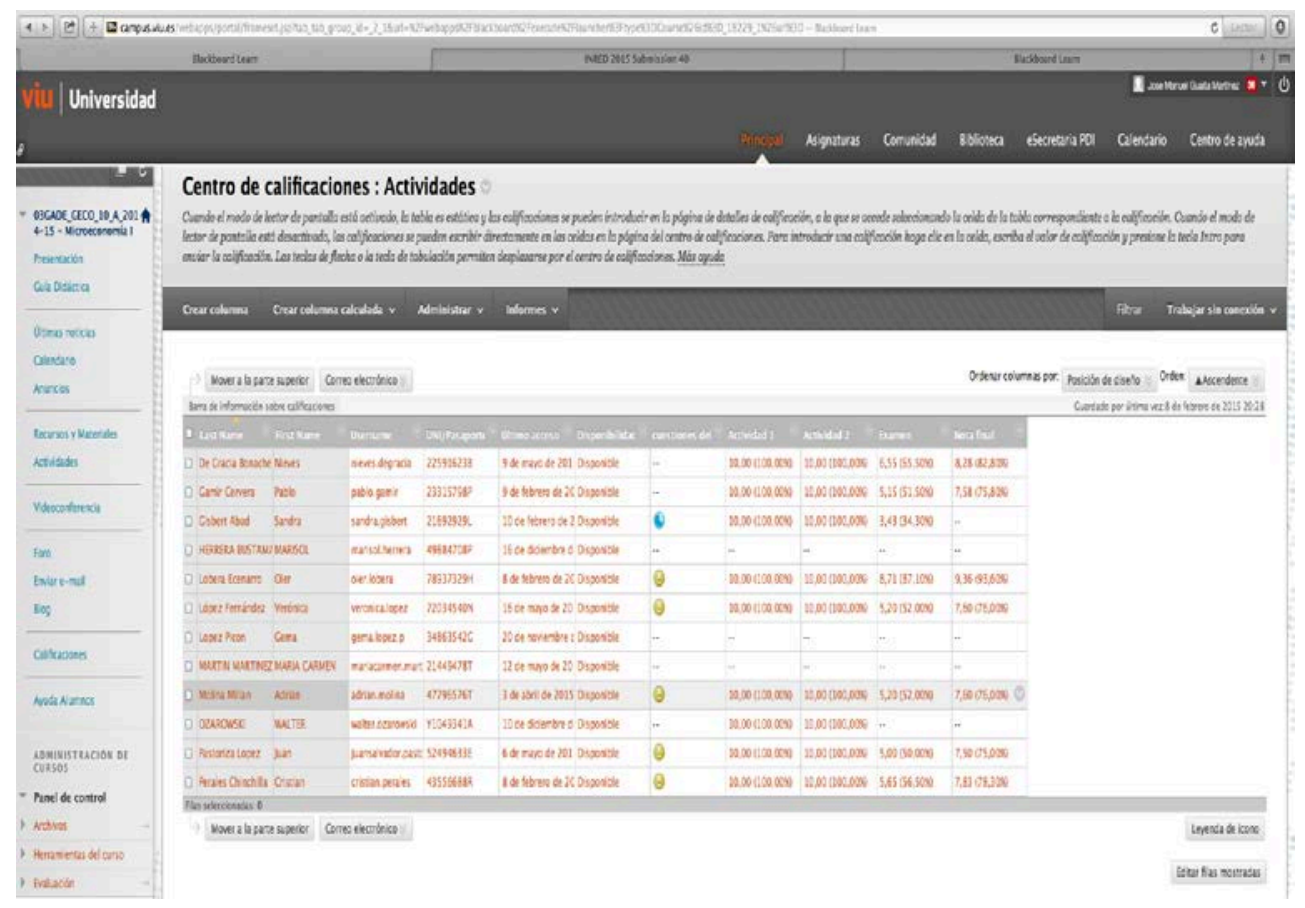

Figura 2. Ejemplo de portfolio de los alumnos en una asignatura.

Se ha analizado la entrega de las actividades guiadas de las 182 asignaturas matriculadas y se han obtenido los siguientes resultados mostrados en la tabla 1. 
Tabla 1. Resultados de las actividades guiadas

\begin{tabular}{|c|c|}
\hline Actividades Solicitadas & $\mathbf{5 4 6}$ \\
\hline Actividades Realizadas en 1a convocatoria & 339 \\
\hline Porcentaje realizadas/solicitadas & $62 \%$ \\
\hline Entregadas en tiempo & $98 \%$ \\
\hline Calificación media de las actividades entregadas & $80 \%$ \\
\hline
\end{tabular}

Estos resultados corresponden a las actividades guiadas relativas a la primera convocatoria, entregándose 339 actividades sobre las 546 totales (182 x 3) siendo entregadas un 98\% de las mismas en el tiempo estipulado y su calificación media un $80 \%$. Cabe destacar que estos resultados corresponden a la evaluación continua, completada con la prueba presencial de cada asignatura.

\section{Conclusiones}

Esta primera experiencia del primer curso de los GE y GADE, en formato exclusivamente online, se está mostrando muy positiva. La valoración del alumnado de las asignaturas impartidas resulta muy provechosa tanto en contenidos, valoración del profesorado, calidad como en la organización de cada una de ellas. Los alumnos han manifestado a lo largo del curso la necesidad de realizar sesiones de repaso una o dos semanas antes de los exámenes como parte de su preparación hacía la realización de las pruebas presenciales en primera y segunda convocatoria.

En el contexto de una formación de Grado online, el alumno es consciente de que está recibiendo una formación de calidad, constituyendo sólo el punto de partida para que, con su participación y esfuerzo personal, desarrolle procesos de mejora e innovación en la práctica profesional. Este perfil requiere la implicación plena del estudiante, se exige una participación activa en las propuestas docentes, pues al finalizar su proceso de aprendizaje, deberá convertirse en el profesional capaz de detectar las necesidades e intervenir sobre ellas.

La principal conclusión es la plena adaptación de los alumnos a las nuevas tecnologías educativas, mostrando un dominio pleno de la herramienta informática utilizada y obteniendo una tasa de éxito cercana al $80 \%$ en las diferentes actividades guiadas que han incluido casos prácticos, visualización de videos y películas, presentación de videos con diapositivas y trabajos de temas concretos.

\section{Referencias}

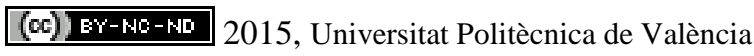

Congreso In-Red (2015) 
Análisis de la implantación del Grado de Economía y Administración y Dirección de Empresas a través de su metodología exclusivamente online.

ESCAMEZ, F. A. N., MUÑOZ, A. D. M. R. y MORENO-MONTOYA, M. (2010). "Un ejemplo de portafolio electrónico para el trabajo en grupo basado en la plataforma WebCT-Blackboard" en RISTI: Revista Ibérica de Sistemas e Tecnologias de Informação, 5, p. 17-30.

GIL, J. J. S. (2015). “Innovación pedagógica en educación continua: hacia un curso de metodología de investigación clínica en el marco de Espacio Europeo de Educación Superior usando la plataforma educativa Blackboard” en RIESED-Revista Internacional de Estudios sobre Sistemas Educativos, 2(4), p. 109-118.

REYES, N. M. y CHAPARRO, F. (2013). «Active methodologies for teaching International Financial Reporting Standards in a virtual learning environment » en Cuadernos de Contabilidad, 14, p. 1147-1182.

BLACKBOARD INC. Manual blackboard collaborate

< $\underline{\text { http://es.blackboard.com/sites/international/globalmaster/Platforms/Blackboard-Collaborate.html }>}$ 